\title{
S-trityl-L-cysteine, a novel Eg5 inhibitor, is a potent chemotherapeutic strategy in neuroblastoma
}

\author{
WEI WU ${ }^{1}$, SHAO JINGBO $^{2}$, WEIJUE XU ${ }^{1}$, JIANGBIN LIU ${ }^{1}$, \\ YIMING HUANG $^{1}$, QINGFENG SHENG ${ }^{1}$ and ZHIBAO LV ${ }^{1}$ \\ Departments of ${ }^{1}$ General Surgery and ${ }^{2}$ Hematology, Shanghai Children's Hospital, \\ Shanghai Jiao Tong University, Shanghai 200062, P.R. China
}

Received March 3, 2017; Accepted February 27, 2018

DOI: $10.3892 / \mathrm{ol} .2018 .8755$

\begin{abstract}
Eg5 is a member of the kinesin-5 family. It is involved in the formation of the bipolar spindle and serves a crucial role in mitosis; meaning that mitotic activation may serve as a chemotherapeutic strategy. However, the anticancer activity of Eg5 inhibitors in neuroblastoma remains uncharacterized. In the present study, the expression of Eg5 was examined in clinical tissue samples and neuroblastoma cell lines, SK-N-SH, SH-SY5Y and SK-N-BE2. Additionally, the antitumor activity of the Eg5 inhibitor, S-trityl-L-cysteine (STLC), was confirmed in vitro. STLC could mediate cell apoptosis, as well as cell cycle arrest, in a dose-dependent manner, which may contribute toward its antitumor activity. STLC-mediated apoptosis and cell cycle arrest were triggered by activation of the mitogen-activated protein kinase and nuclear factor $\mathrm{kB}$ signaling pathways. These results suggested that STLC may have potential in the in vivo treatment of neuroblastoma.
\end{abstract}

\section{Introduction}

Neuroblastoma (NB) is among the most common types of solid tumor in children (1). NB has a high degree of malignancy, a high mortality rate and a poor prognosis, posing diagnostic and therapeutic challenges for pediatricians $(2,3)$. Spontaneous remission occurs in the majority of infants with NB $(3,4)$. However, the disease may be disseminated to the liver, skin and bone marrow at diagnosis (1). The survival rate of infants with stage $4 \mathrm{~s}$ NB $>95 \%(2,3)$. Children with high-risk NB are resistant to intensive therapy, and the prognosis for recurrent or metastatic disease is poor, with a 5-year survival rate of $<30 \%$ (5). Therefore, the identification of

Correspondence to: Dr Zhibao Lv, Department of General Surgery, Shanghai Children's Hospital, Shanghai Jiao Tong University, 355 Lu Ding Road, Shanghai 200062, P.R. China

E-mail:1vzhibao@sohu.com

Key words: Eg5 inhibitor, S-trityl-L-cysteine, neuroblastoma, apoptosis effective and efficient targeted therapies is required to improve the prognosis of patients with NB.

It has been reported that MYCN gene amplification occurs in $25-30 \%$ of NB cases, and that it is a predictive biomarker of a poor prognosis and tumor aggressiveness (3). Eg5, also known as kinesin-like spindle protein, is a member of the mitotic kinesin superfamily, which modulates microtubule tracks for intracellular transport or cell division (6). A number of Eg5 inhibitors have undergone clinical testing as antimitotic drugs (7-11). Minstrel and its analogs, as well as other chemically distinct small molecules, including S-trityl L-cysteine (STLC), are allosteric inhibitors that bind to a unique pocket in the Eg5 motor domain formed by secondary structural elements (helix a2/loop L5/helix a3) $(12,13)$.

In the present study, Eg5 expression was examined in human NB SK-N-SH, SH-SY5Y and SK-N-BE2 cell lines, and tissue specimens from patients with NB using immunofluorescence and western blotting. It has been reported that the antitumor activity of Eg5 inhibitors depends on cell cycle arrest and the promotion of cell apoptosis (7). However, the underlying molecular mechanisms remain to be elucidated.

\section{Materials and methods}

Materials. The human NB SH-SY5Y (SY5Y), SK-N-SH (SK) and SK-N-BE2 (BE2) cell lines were purchased from The Cell Bank of Type Culture Collection of Chinese Academy of Sciences (Shanghai, China). The cells were maintained in a humidified atmosphere containing $5 \% \mathrm{CO}_{2}$ and $95 \%$ air at $37^{\circ} \mathrm{C}$ in Dulbecco's modified Eagle's medium (DMEM; Gibco; Thermo Fisher Scientific, Inc., Waltham, MA, USA), supplemented with $10 \%$ fetal bovine serum (FBS; Gibco; Thermo Fisher Scientific, Inc.). The anti-Eg5 (cat. no. ab51976; 1:400) and anti- $\beta$-actin (ab6276 1;250) primary antibodies were obtained from Abcam (Cambridge, UK). The goat anti-mouse Alexa Fluor 647 (cat. no. ab150119; 1:250) was purchased from Jackson ImmunoResearch Europe, Ltd. (Newmarket, UK). STLC was obtained from Sigma-Aldrich; Merck KGaA (Darmstadt, Germany). The apoptosis detection kit [BD Pharmingen-fluorescein isothiocyanate (FITC) Annexin V Apoptosis Detection kit; cat no. 556547] and Cell Cycle kit (BD Cycletest ${ }^{\mathrm{TM}}$ Plus; cat no. 340242) were purchased from BD Biosciences (Franklin Lakes, NJ, USA). 
Specimen information. The Institutional Review Board of Shanghai Children's Hospital (Shanghai, China) approved the study protocol and waived the requirement for informed consent (2015-02-11, no. 1). The NB and GNB tissue specimens were collected from 3 patients with NB or GNB (16.7 \pm 6.43 months). In order to verify the expression in different tissues, the experiments were also performed in normal tissues without $\mathrm{NB}$, including renal (collected from patient with Wilms' tumor upon nephrectomy) and liver (collected from patients with Choledochal cysts). The control tissues were obtained from the Pathology Department of Shanghai Children's Hospital (Shanghai China), and were retrospectively analyzed. All diagnoses were confirmed pathologically. Clinical information is reported in Table I.

Immunofluorescence. Immunofluorescence staining was utilized to detect the expression of Eg5 in NB cell lines. Cells were plated onto glass coverslips and allowed to attach in order to obtain a sufficient number of cells to perform immunofluorescence. SY5Y, SK and BE2 cells were fixed with $3 \%$ paraformaldehyde and permeabilized with $0.15 \%$ Triton X-100 for $12 \mathrm{~h}$ at $4^{\circ} \mathrm{C}$. The primary antibodies (1:200) were incubated with the slides at room temperature for $1 \mathrm{~h}$. An Alexa Fluor 488 secondary antibody was added for $45 \mathrm{~min}$ at room temperature. DAPI $(1 \mu \mathrm{g} / \mathrm{ml}$; Beyotime Institute of Biotechnology, Haimen, China) was used to stain the nuclei for $20 \mathrm{~min}$ at room temperature.

Western blotting. SY5Y, SK or BE2 cells were lysed using radioimmunoprecipitation assay buffer (Thermo Fisher Scientific, Inc.) containing protease and phosphatase inhibitors (Thermo Fisher Scientific, Inc.). Protein concentration was measured using a bicinchoninic acid protein assay kit (Thermo Fisher Scientific, Inc.), and equal amounts (30 $\mu \mathrm{g})$ of protein from each cell lysate were solubilized in $2 \mathrm{X}$ SDS loading buffer (Beyotime Institute of Biotechnology, Haimen, China), then separated via $15 \%$ SDS-PAGE and transferred into nitrocellulose membranes. The membranes were blocked in 5\% bovine serum albumin (Gibco; Thermo Fisher Scientific, Inc.), and then probed with anti-Eg5 (1:400; cat no. ab51976; Abcam, Cambridge, USA) and $\beta$-actin (1:5,000; AF003; Beyotime Institute of Biotechnology) overnight at $4^{\circ} \mathrm{C}$ and subsequent hybridization with a HRP-conjugated goat anti-mouse secondary antibody (1:500; cat.no. 115035003; Jackson ImmunoResearch Europe, Ltd.) for $1 \mathrm{~h}$ at room temperature. Following 3 washes with Tris-buffered saline with Tween, the membranes were scanned using aLAS-4000 Mini system (FujiFilm, Tokyo, Japan).

Flow cytometric analysis of apoptosis. Cells were seeded into a 12 -well plate and treated with $0,1,5,10$ or $20 \mu \mathrm{mol} / 1$ STLC for $72 \mathrm{~h}$. The cells were then harvested by trypsin and washed with PBS. The cells were stained with $5 \mu \mathrm{l}$ Annexin $\mathrm{V}$ and $5 \mu 17-\mathrm{AAD} 1 \mathrm{~h}$ at $37^{\circ} \mathrm{C}$ using a BD Pharmingen-FITC Annexin V Apoptosis Detection kit (cat. no. 556547; BD Biosciences), according to the manufacturer's protocol. Apoptotic cells were analyzed using a BD Fortessa flow cytometer (BD Biosciences). The data were analyzed using FlowJo software LLCv.10 (Ashland, OR, USA).
Flow cytometric analysis of cell cycle. A total of $3 \times 10^{4}$ cells were seeded into a 12-well plate and treated with $1,5,10$ or $20 \mu \mathrm{mol} / 1 \mathrm{STLC}$ for $72 \mathrm{~h}$. The cells were then harvested and washed with PBS. The cells were fixed and treated with $10 \%$ propidium iodide at $37^{\circ} \mathrm{C} 2 \mathrm{~h}$ (BD Biosciences), according to the manufacturer's protocol. Cells were analyzed using a BD Fortessa flow cytometer (BD Biosciences). The percentage of cells in the G1, S and G2-M phases was calculated. All experiments were performed in triplicate. The data were analyzed using FlowJo software LLCv.10 (Ashland, OR, SA).

Fluorescence in situ hybridization (FISH). A total of $3 \times 10^{4}$ cells (SK, SY5Y and BE2) were seeded into a 6-well plate. The cells were then harvested by trypsin and washed with PBS. BE2 were seeded into a 6-well plate treated with $1 \mu \mathrm{mol} / 1$ or $5 \mu \mathrm{mol} / 1 \mathrm{STLC}$ for $48 \mathrm{~h}$. Vysis LIS N-MYC SO Probe (Abbott Pharmaceutical Co., Ltd., Lake Bluff, IL, USA) with its corresponding hybridization buffer was removed, $1 \mu \mathrm{l}$

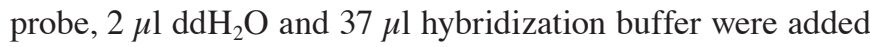
to make up a final volume of $40 \mu \mathrm{l}$. The solution was microcentrifuged for $2 \mathrm{sec}$ at $37^{\circ} \mathrm{C}$ at $550 \mathrm{x} \mathrm{g}, 10 \mu \mathrm{l}$ probe was added onto the slide and a square glass coverslip was added for $2 \mathrm{~min}$ at $72^{\circ} \mathrm{C}$. The solution was placed into a humidified chamber at $37^{\circ} \mathrm{C}$ overnight. A coplin jar containing $40 \mathrm{ml} 0.4 \mathrm{X} \mathrm{SSC}$ was placed in a $75^{\circ} \mathrm{C}$ water bath for $30 \mathrm{~min}$. A total of $40 \mathrm{ml}$ 2X SSC/0.1\% NP40 was added to another coplin jar at room temperature. Slides were removed from the incubation chamber and rubber cement and coverslips from the first 4 slides were removed. The slides were placed directly into $2 \mathrm{X} \mathrm{SSC} / 0.1 \%$ $\mathrm{NP} 40$ for $1 \mathrm{~min}$ at $37^{\circ} \mathrm{C}$. Slides were removed and dried off using a paper towel. Slides were air dried in the dark. A total of $10 \mu \mathrm{l}$ DAPI II was applied at room temperature for $1 \mathrm{~h}$, prior to being transferred to a $24 \times 50 \mathrm{~mm}$ coverslip. Epi-fluorescence microscopy was then used at $\mathrm{x} 1,000$ magnification.

mRNA microarray analysis. SY5Y were seeded into a 6-well plate treated with 0 or $5 \mu \mathrm{mol} / 1 \mathrm{STLC}$ for $72 \mathrm{~h}$ at $37^{\circ} \mathrm{C}$, the cells were then harvested by trypsin and washed twice with PBS. Total RNA was isolated using TRIzol ${ }^{\circledR}$ reagent (Thermo Fisher Scientific, Inc.), according to the manufacturer's protocols, and purified using an RNeasy Mini kit (Qiagen $\mathrm{GmbH}$, Hilden, Germany). RNA samples were processed for array hybridization using the GeneChip one-cycle target labeling kit (Affymetrix, Inc, Santa Clara, CA, USA) for amplification and labeling of total RNA. Target synthesis was performed following the Affymetrix GeneChip Expression Analysis Technical Manual, rev. 5 (http://www.affymetrix. com/support/technical/manual/expression_manual.affx) with minor modification. Using 2-4 $\mu \mathrm{g}$ total RNA as an input, mRNA was converted to double-stranded cDNA and purified by phenol-chloroform-isoamyl alcohol extraction and ethanol precipitation to generate biotinylated cRNA targets and then biotinylated cRNA targets for the GeneChip ${ }^{\circledR}$ PrimeView ${ }^{\mathrm{TM}} / \mathrm{U} 133$ plus 2.0 Human Gene Expression Array. The biotinylated cRNA targets were then hybridized with the microarray. Following hybridization, arrays were stained with Cy5-dCTP in the Fluidics Station 450 at $45^{\circ} \mathrm{C}$ for $16 \mathrm{~h}$ and scanned on the Affymetrix Scanner 3000. The microarray experiments were performed according to the protocol of Affymetrix (Thermo Fisher Scientific, Inc.). The raw data 
Table I. Clinical information of tissue specimens.

\begin{tabular}{|c|c|c|c|c|c|c|}
\hline Name & $\begin{array}{l}\text { Age at the time of } \\
\text { diagnosis (months) }\end{array}$ & Sex & Diagnosis & $\begin{array}{c}\text { Date of } \\
\text { collection }\end{array}$ & Pathology & Tissues \\
\hline NB1 & 12 & Male & NB III & June, 2014 & NB & Tumor \\
\hline NB2 & 14 & Female & NB III & August, 2015 & NB & Tumor \\
\hline GNB & 24 & Male & GNB II & October, 2014 & GNB & Tumor \\
\hline Liver intestine & 22 & Male & Choledochal cyst & October 2015 & Choledochalcyst & $\begin{array}{l}\text { Normal liver } \\
\text { intestine }\end{array}$ \\
\hline $\begin{array}{l}\text { Renal tubule } \\
\text { Glomerulus }\end{array}$ & 9 & Male & Wilms' tumor & November, 2015 & Wilms'tumor & $\begin{array}{l}\text { Normal renal } \\
\text { tissue }\end{array}$ \\
\hline
\end{tabular}

NB, Neuroblastoma; GNB, ganglioneuroblastoma.
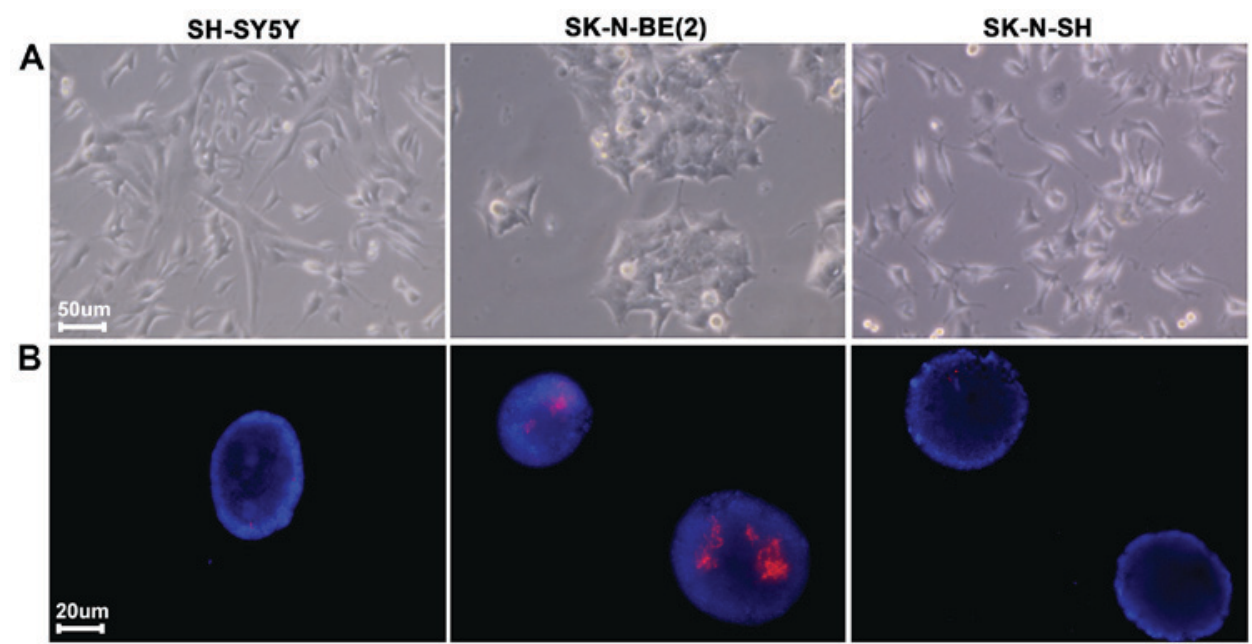

Figure 1. Neuroblastoma MYC oncogene amplification detection in SH-SY5Y, SK-N-BE2 and SK-N-SH cells by fluorescence in situ hybridization analysis. (A) Cell morphology and (B) fluorescence in situ hybridization analysis where red-MYCN probe blue-DAPI. MYC, myelocytomatosis viral-related oncogene.

were normalized with the MAS 5.0/RMA algorithm to the control group using the Gene Spring Software 11.0 (Agilent Technologies, Inc., Santa Clara, CA, USA). Genes with a fold change of $>2$ were selected for further analysis.

Statistical analysis. All statistical analyses were performed using GraphPad Prism 5 software (GraphPad Software, Inc., La Jolla, CA, USA). Data are expressed as the mean \pm standard deviation. Comparisons of the means of 2 groups were conducted using Student's t-test. Comparisons of the means of $\geq 3$ groups were conducted using one-way analysis of variance. Comparisons between the groups was made by analyzing data with Student-Newman-Keuls post hoc method. $\mathrm{P}<0.05$ was considered to indicate a statistically significant difference.

\section{Results}

Expression level of MYC oncogene (MYCN) in NB cells. Previous studies have demonstrated that amplification and overexpression of MYCN is associated with a poor prognosis in NB $(2,3)$. To investigate and confirm the expression of MYCN in SK, SY5Y and BE2 cell lines, MYCN gene amplification was detected by fluorescence in situ hybridization
(FISH). MYCN gene amplification was identified in BE2 cells (Fig. 1).

Eg5 in NB tissues specimens and cell lines. Eg5 is expressed in the testis, thymus, tonsils and bone marrow and is absent from the adult human central nervous system. Eg5 is overexpressed in breast, lung, ovarian, bladder and pancreatic cancer. Previous studies have demonstrated that Eg5 is of central importance in driving the assembly of the mitotic spindle, and is also a promising chemotherapeutic target. Inhibition of Eg5 by small molecule inhibitors results in monopolar spindles and mitotic arrest, which may lead to cell death $(14,15)$. However, to the best of our knowledge, no previous studies have reported whether Eg5 is expressed in NB. Therefore, in the present study, the expression level of Eg5 was determined in SK, SY5Y and BE2 cell lines (Fig. 2A and B). Eg5 protein expression in NB and NGB tissues was observed using immunofluorescence (Fig. 2C). Eg5 staining was also observed in the glomerulus but not in liver, renal tubule or intestine tissues (Fig. 2D).

STLC has no effect on MYCN gene amplification and expression. As a selective allosteric inhibitor of Eg5, STLC blocked the bipolar spindle formation that causes mitotic arrest 


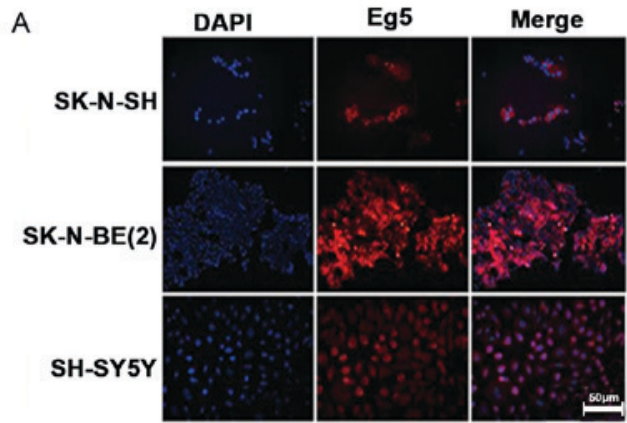

B
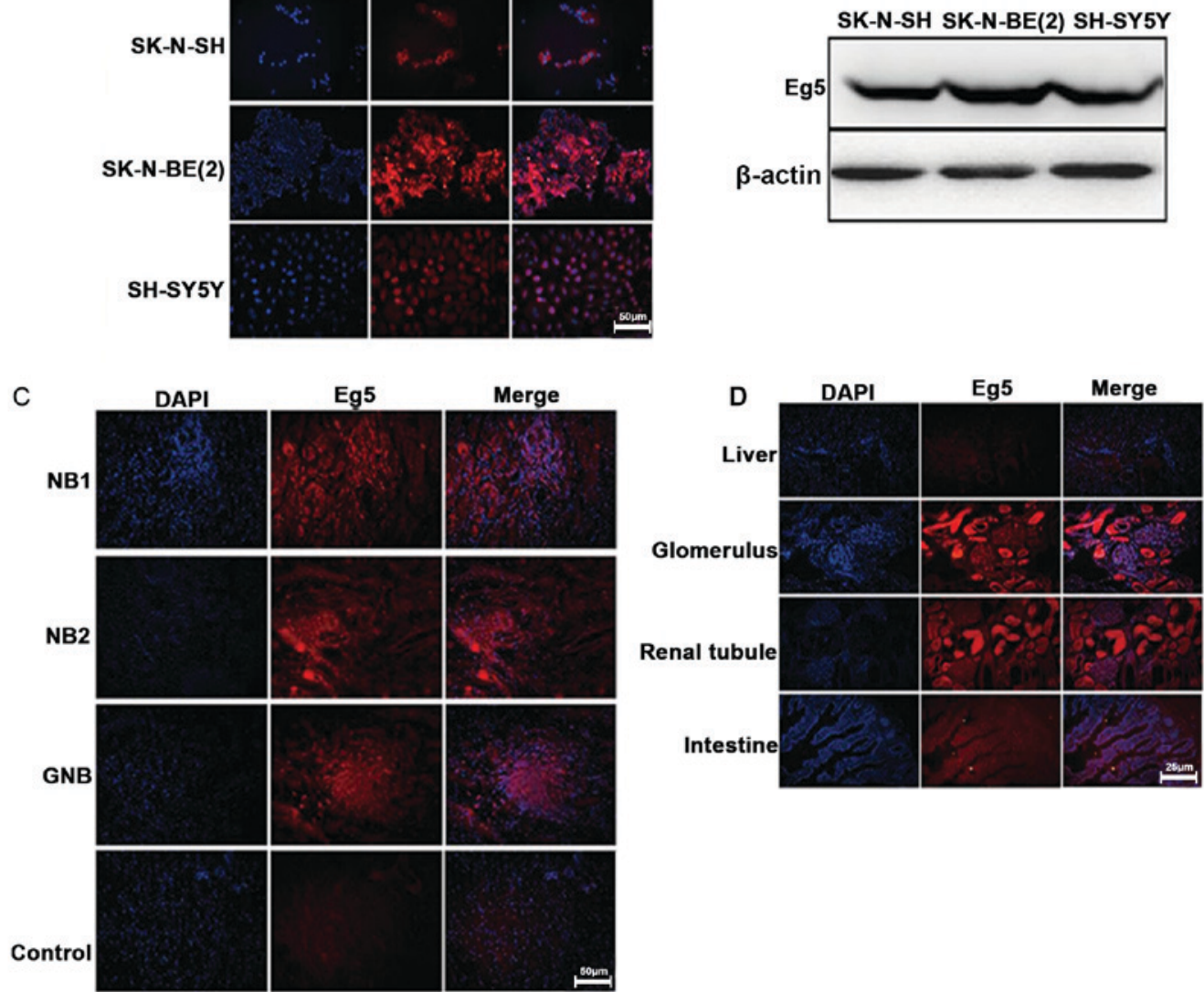

Figure 2. Immunofluorescence and western blot analysis of Eg5 expression in NB tissues and cell lines. (A) Immunofluorescence analysis of Eg5 protein expression in NB cell lines. (B) Western blotting illustrated expression of Eg5 in NB cell lines. (C) Immunofluorescence analysis of Eg5 protein expression in NB and GNB specimens. (D) Immunofluorescence analysis of Eg5 protein expression in different tissues without NB and GNB. Scale bar, 25-50 $\mu \mathrm{m}$. NB, neuroblastoma; GNB, ganglioneuroblastoma.
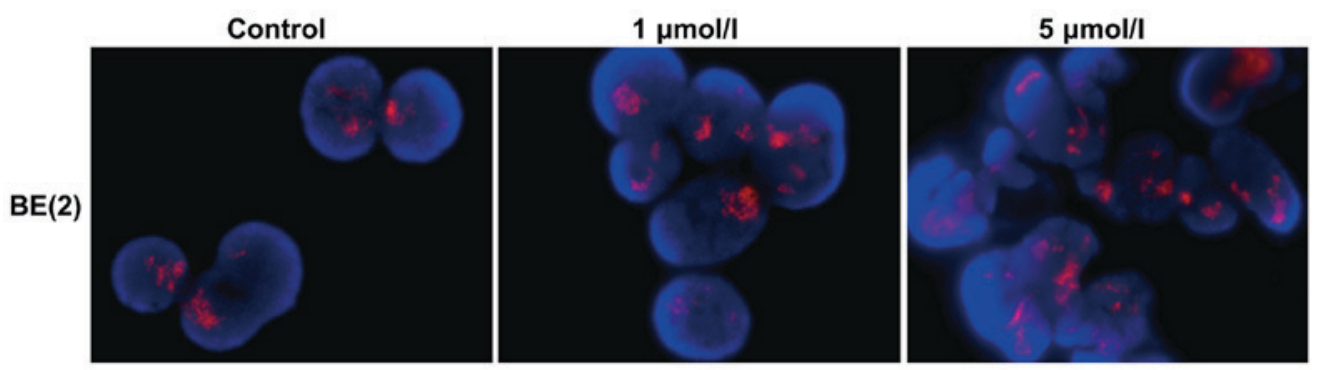

Figure 3. MYCN expression in BE2 cells treated with STLC for $48 \mathrm{~h}$. BE2 cells were treated with 1 or $5 \mu \mathrm{mol} / 1 \mathrm{STLC}$ for $48 \mathrm{~h}$. MYCN gene level was detected by fluorescence in situ hybridization. There was no evident difference in MYCN expression among these groups. MYCN, myelocytomatosis viral-related oncogene neuroblastoma derived; STLC, S-trityl- L-cysteine.

and ultimately leads to apoptotic cell death (7). In order to investigate whether STLC affects MYCN expression, the BE2 cells were treated in the absence or presence of the inhibitor ( 1 and $5 \mu \mathrm{mol} / 1 \mathrm{STLC}$ for $48 \mathrm{~h}$ due to the differences observed at these concentrations). STLC treatment did not affect MYCN amplification (Fig. 3), suggesting that the antitumor activity of STLC was not dependent upon MYCN amplification.

STLC inhibits the expression of Eg5 protein and promotes cell apoptosis and cell cycle arrest. STLC is known to cause cell death and inhibit cell proliferation in solid tumors (11). The present study aimed to determine whether STLC may induce apoptosis and cell cycle arrest in NB cells. The percentage of apoptotic cells in the SY5Y and BE2 cell lines increased with increased STLC concentrations (Fig. 4A). Compared with the untreated control group, the change in the percentage of apoptotic cells was notable at $5 \mu \mathrm{mol} / 1 \mathrm{STLC}$. Flow cytometric analysis indicated that STLC treatment induced cell cycle arrest at G2/M phase (Fig. 4B). In accordance with the cell apoptosis results, the population shift in cell cycle arrest from G1 phase to G2/M phase was notable at $5 \mu \mathrm{mol} / 1 \mathrm{STLC}$. The expression of Eg5 decreased in a dose-dependent manner in response to STLC treatment (Fig. 4C) with no marked differences between the 48 and $72 \mathrm{~h}$ time points. 

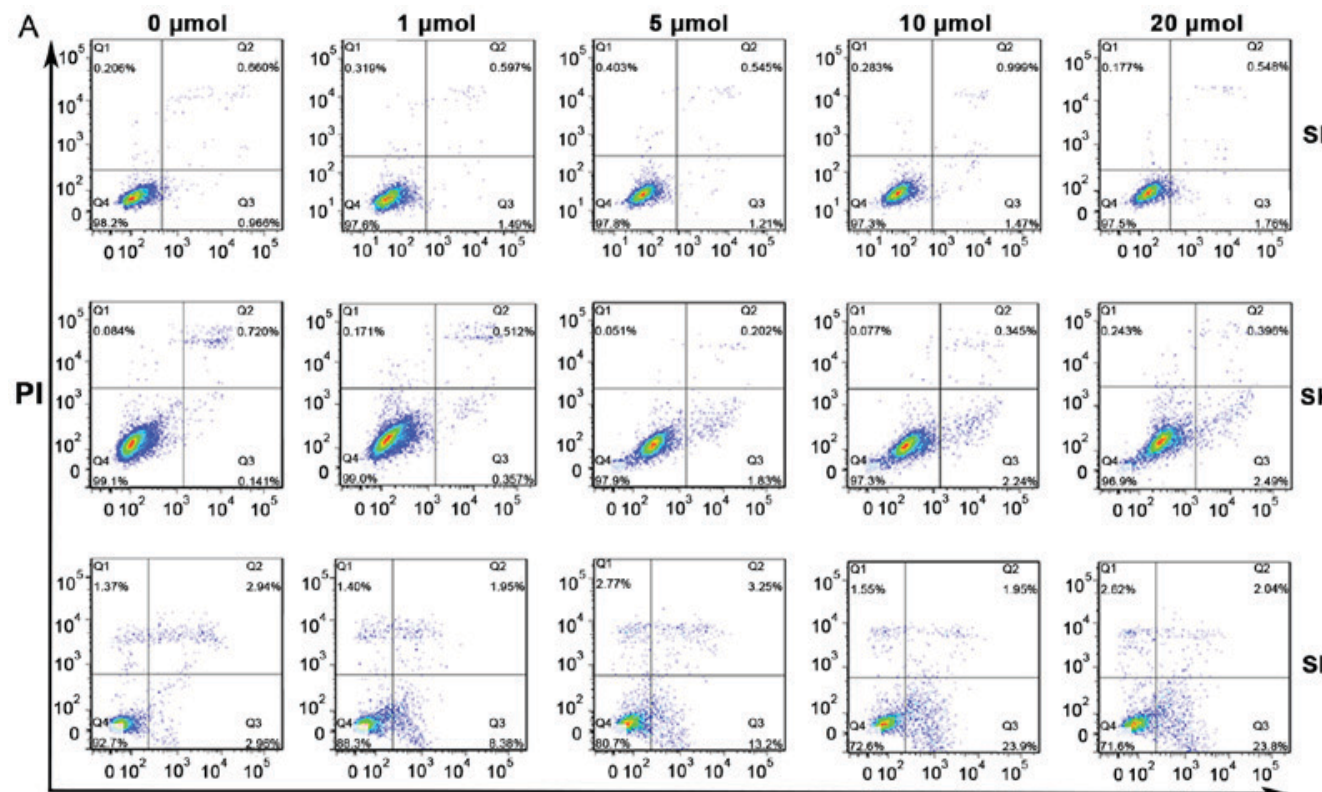

SK-N-SH
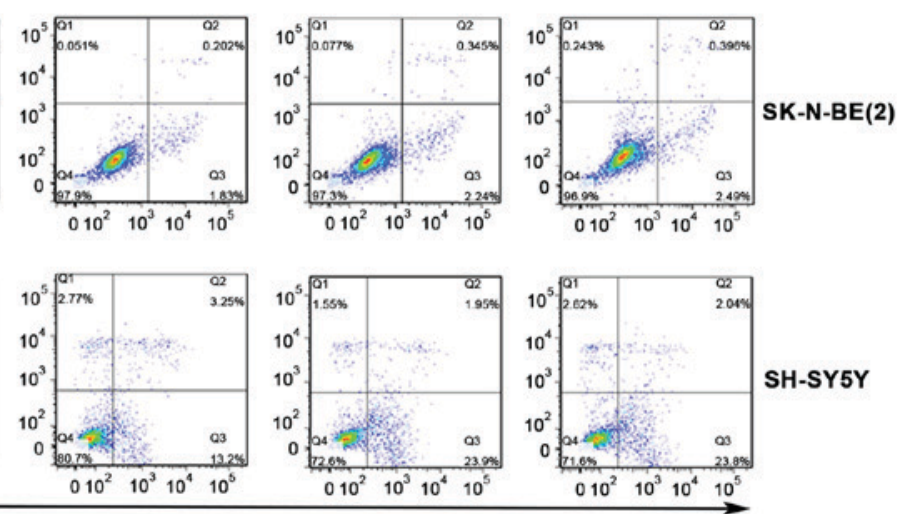

SH-SY5Y

Annexin V-7AAD

B
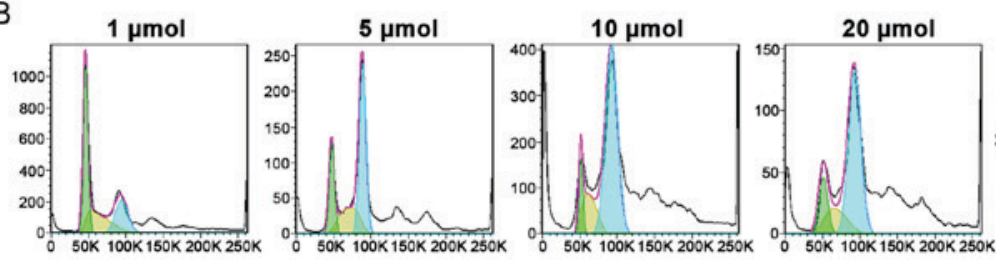

SK-N-SH
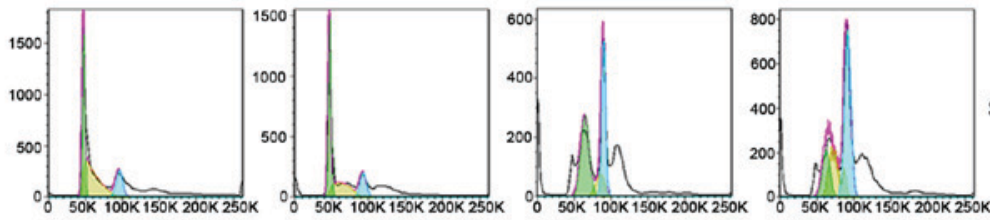

SK-N-BE(2)
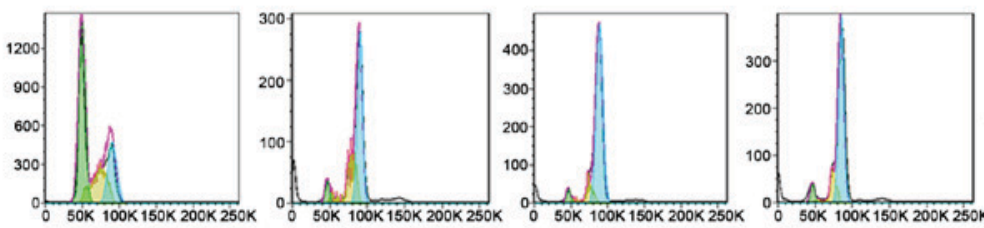

SH-SY5Y

PI

\section{C}

STLC stimulation for $48 \mathrm{~h}$

STLC stimulation for $72 \mathrm{~h}$
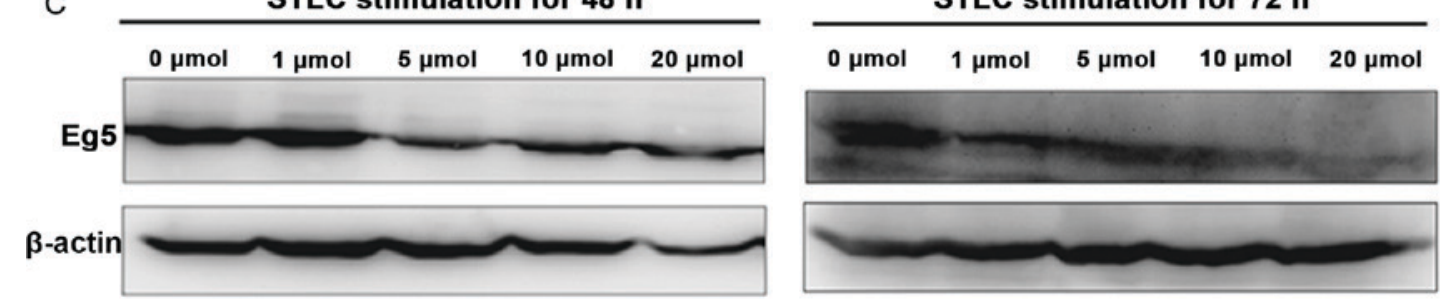

Figure 4. The effect of STLC treatment on the expression of Eg5, cell apoptosis and cell cycle progression in NB. SK, SY5Y and BE2 cells were treated with $0,1,5,10$ and $20 \mathrm{mmol} \mathrm{STLC}$ for $72 \mathrm{~h}$. (A) Flow cytometric analysis of the cell apoptosis. The percentage of apoptotic cells was dose-dependent in the NB cells (B) The effect of STLC on the progression of the cell cycle in NB cells. The amplitude of curves corresponds to the cell number, the peak on the left (green) represents cells in the G1 phase of the cell cycle, while the peak on the right (blue) represents cells in the G2/M phase. Compared with cells treated with1 mmol STLC, the percentage of G2/M phase was significantly increased in higher concentrations. (C) Western blotting revealed that STLC treatment of SY5Y cells for 48 and $72 \mathrm{~h}$ resulted in a decrease of Eg5 expression. STLC, S-trityl- L-cysteine; NB, neuroblastoma; PI, propidium iodine.

mRNA microarray analysis. GSEA analysis demonstrated that STLC regulates MAPK and NF- $\kappa B$ signaling pathways. The results demonstrated that the MAPK and $\mathrm{NF}-\kappa \mathrm{B}$ signaling pathways were activated in cells treated with $5 \mu \mathrm{mol} / 1$ STLC compared with the control group (Fig. 5). 

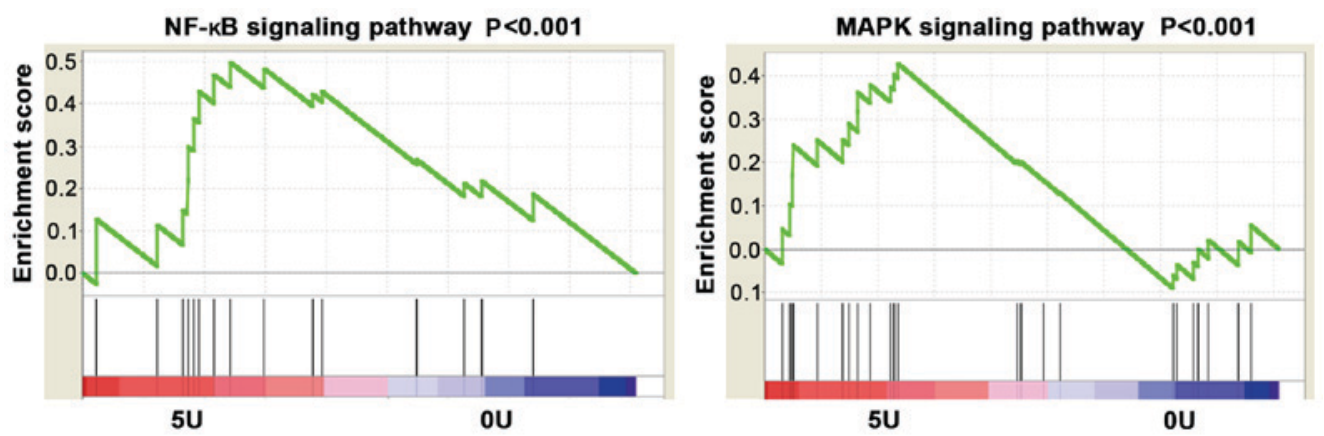

Figure 5. Enrichment plots of gene expression signatures for NF- $\mathrm{kB}$ and MAPK signaling pathways. SY5Y cells were stimulated with 0 and $5 \mu \mathrm{mol} / 1$ STLC for $72 \mathrm{~h}$. Gene-set enrichment analysis of the NF- $\mathrm{KB}$ and MAPK signaling pathways in SY5Y-treated cells. The green line represents enrichment score (GSEA gene set enrichment analysis), red represents more enrichment and blue represents less enrichment. STLC, S-trityl- L-cysteine; NF- $\mathrm{B}$, nuclear factor $\kappa B$; MAPK, mitogen-activated protein kinase; $5 \mathrm{U}, 5 \mu \mathrm{mol} / 1 ; 0 \mathrm{U}, 0 \mu \mathrm{mol} / \mathrm{l}$.

\section{Discussion}

NB is a pediatric disease, which arises from sympathoadrenergic neural crest progenitor cells of the peripheral nervous system $(16,17)$. Due to the high risk and recurrence rate of advanced NB, and the lack of specific biomarkers, there are currently no efficient treatment approaches for neuroblastoma (18). Methods for predicting patient response to chemotherapy are being developed (3). The present study aimed to identify the signaling pathways associated with STLC.

Previous research has suggested that promoting cell apoptosis and cell cycle progression arrest may be an important molecular mechanism of antitumor activity $(17,18)$. In the present study, three NB cell lines (SY5Y, SK and BE2) were used to examine STLC-induced cell apoptosis and cell cycle progression.

As a kinesin spindle protein, $\mathrm{Eg} 5$ is involved in mitosis and its inhibition promotes mitotic arrest $(19,20)$. Eg5 inhibitors are effective compared with anti-tubulin drugs, which have dose-limiting side effects (21). Eg5 does not serve a role in resting and non-dividing cells (13). Beneficial effects of suppression of Eg5 function/expression have been suggested in breast, lung, colon, ovary and prostate cancer in vitro (22-24).

Numerous inhibitors of Eg5 have been discovered, including monastrol, S-trityl-L-cysteine (STLC) and ispinesib (7). These inhibitors bind an allosteric site located between a helix 3 and loop 5 of the Eg5 domain (13). Although Eg5 inhibitors have reached pre-clinical dose-limiting toxicity trials, the molecular mechanism underlying the antitumor activity has not been elucidated (25). EMD 534085, a potent, reversible Eg5 inhibitor, has demonstrated significant preclinical antitumor activity (10). Previous studies reported that a selective inhibitor, LY2523355, arrests cancer cells in mitosis and causes rapid cell death $(8,10,26)$. Previous research has evaluated the function of STLC in prostate cancer, indicating that docetaxel-resistant prostate cancer cells remain responsive to Eg5 inhibition in response to STLC treatment $(12,27)$. STLC has been demonstrated to exhibit higher potency compared with monastrol or terpendole $\mathrm{E}$ in inducing mitotic arrest (28).

In the present study, the anticancer activity of STLC was characterized in NB. Eg5 was demonstrated to be overexpressed in NB tissue specimens and cell lines. It was also observed that the expression level of Eg5 was increased in
BE2 cells exhibiting MYCN amplification compared with SY5Y and SK cells. Therefore, it was hypothesized that the expression of MYCN, an essential regulator of cancer progression (3), is associated with the antitumor activity of STLC. However, immunofluorescence analysis revealed that STLC did not affect the expression of MYCN. To further investigate whether STLC exhibits antitumor activity in NB, cell apoptosis and cell cycle progression was analyzed. Cell cycle analysis demonstrated that STLC treatment resulted in a dose-dependent increase in the number of cells with polyploidy DNA content, and that the cell cycle was arrested at G2/M phase. Cell apoptosis analysis revealed that STLC induced cell apoptosis in a dose-dependent manner.

Centrosome separation is stringently controlled by mitotic kinases, including cyclin-dependent kinase 1 (Cdk1), polo-like kinase 1, Aurora A and mitosis gene-A-related kinase 2. However, the molecular mechanism by which these kinases contribute toward the activity of Eg5 inhibitors remains unknown $(29,30)$. Previous studies have identified that Cdk1 triggers centrosome separation in the late $\mathrm{G} 2$ phase by phosphorylating the motor protein Eg5 at Thr927 $(31,32)$. In the present study, a robust increase in the percentage of apoptotic and G2/M-phase DNA content was observed in response to $5 \mu \mathrm{mol} / 1$ STLC compared with control cells. At the transcriptional level, a significant activation of the NF- $\mathrm{KB}$ and MAPK pathways was identified at $5 \mu \mathrm{mol} / 1$ STLC compared with control cells. Neuronal differentiation in early development involves neuronal fate determination and a series of morphological changes, including neurite initiation, extension, and maturation of axons and dendrites (33). During neurite growth, multiple signaling pathways are activated, including the MAPK and PI3K-Akt pathways (34). Whether or not the activation of NF- $\mathrm{KB}$ and MAPK pathways in response to the STLC treatment was associated with neuronal differentiation remains to be elucidated.

To investigate the possible signaling pathways associated with STLC-induced apoptosis, the activity, expression and localization of the caspase $3 / 8$ were analyzed. It has been previously investigated how anti-mitotic drugs cause apoptosis. A number of studies have suggested that the effectiveness of the spindle checkpoint is the primary determinant in response to taxanes and other anti-mitotic drugs (35-37). Future studies may involve determining the apoptotic signaling pathway by 
mRNA expression profiling in order to confirm which genes or targets from these signaling pathways significantly affect cell cycle progression.

To the best of our knowledge, the present study was the first to demonstrate cell apoptosis and cell cycle arrest in NB cell lines induced by STLC. The investigation of the NF- $\mathrm{NB}$ and MAPK signaling pathways provides a basis for elucidating the molecular mechanism of STLC antitumor, as well as for the development of therapeutic strategies targeting specific biomarkers in NB.

\section{Acknowledgements}

Not applicable.

\section{Funding}

The present study was supported by a grant from the Shanghai Science and Technology Committee (grant no. 12DZ2295006).

\section{Availability of data and materials}

All data generated or analysed during this study are included in this published article.

\section{Authors' contributions}

WW and SJ conducted experiments, acquired and analysed the datasets. WX and JL were responsible for tissue collection. QS and YH contributed toward analysing and interpreting datasets. ZL conceived and designed the study, secured the funding and gave final approval of the version to be published. All authors read and approved the final manuscript.

\section{Ethics approval and consent to participate}

The Institutional Review Board of Shanghai Children's Hospital (Shanghai, China) approved the study protocol and waived the requirement for informed consent (2015-02-11, approval no. 1).

\section{Consent for publication}

Not applicable.

\section{Competing interests}

The authors declare that they have no competing interests.

\section{References}

1. Hoy SM: Dinutuximab: A review in high-risk neuroblastoma. Target Oncol 11: 247-253, 2016.

2. Brodeur GM: Neuroblastoma: Biological insights into a clinical enigma. Nat Rev Cancer 3: 203-216, 2003.

3. Sano H, Bonadio J, Gerbing RB, London WB, Matthay KK, Lukens JN and Shimada H: International neuroblastoma pathology classification adds independent prognostic information beyond the prognostic contribution of age. Eur J Cancer 42: 1113-1119, 2006.

4. Kraal K, Blom T, Tytgat L, van Santen H, van Noesel M, Smets A, Bramer J, Caron H, Kremer L and van der Pal H: Neuroblastoma with intraspinal extension: Health problems in long-tterm survivors. Pediatr Blood Cancer 63: 990-996, 2016.
5. Gigliotti AR, De Ioris MA, De Grandis E, Podda M, Cellini M, Sorrentino S, De Bernardi B, Paladini D and Gandolfo C: Congenital neuroblastoma with symptoms of epidural compression at birth. Pediatr Hematol Oncol 33: 94-101, 2016.

6. Yokoyama H, Sawada J, Katoh S, Matsuno K, Ogo N, Ishikawa Y, Hashimoto H, Fujii S and Asai A: Structural basis of new allosteric inhibition in kinesin spindle protein Eg5. ACS Chem Boil 10: 1128-1136, 2015.

7. El-Nassan HB: Advances in the discovery of kinesin spindle protein (Eg5) inhibitors as antitumor agents. Eur J Med Chem 62: 614-631, 2013.

8. Wakui H, Yamamoto N, Nakamichi S, Tamura Y, Nokihara H, Yamada Y and Tamura T: Phase 1 and dose-finding study of patritumab (U3-1287), a human monoclonal antibody targeting HER3, in Japanese patients with advanced solid tumors. Cancer Chemother Pharmacol 73: 511-516, 2014.

9. Gerecitano JF, Stephenson JJ, Lewis NL, Osmukhina A, Li J, Wu K, You Z, Huszar D, Skolnik JM and Schwartz GK: A Phase I trial of the kinesin spindle protein (Eg5) inhibitor AZD4877 in patients with solid and lymphoid malignancies. Invest New Drugs 31: 355-362, 2013.

10. Hollebecque A,Deutsch E, Massard C,Gomez-Roca C,Bahleda R, Ribrag V, Bourgier C, Lazar V, Lacroix L, Gazzah A, et al: A phase I, dose-escalation study of the Eg5-inhibitor EMD 534085 in patients with advanced solid tumors or lymphoma. Invest New Drugs 31: 1530-1538, 2013.

11. Ishikawa K, Tohyama K, Mitsuhashi S and Maruta S: Photocontrol of the mitotic kinesin Eg5 using a novel S-trityl-1-cysteine analogue as a photochromic inhibitor. J Biochem 155: 257-263, 2014.

12. Kaan HY, Weiss J, Menger D, Ulaganathan V, Tkocz K, Laggner C, Popowycz F, Joseph B and Kozielski F: Structure-activity relationship and multidrug resistance study of new S-trityl-L-cysteine derivatives as inhibitors of Eg5. J Med Chem 54: 1576-1586, 2011.

13. Ishikawa K, Tamura Y and Maruta S: Photocontrol of mitotic kinesin Eg5 facilitated by thiol-reactive photochromic molecules incorporated into the loop L5 functional loop. J Biochem 155: 195-206, 2014.

14. Sun D, Lu J, Ding K, Bi D, Niu Z, Cao Q, Zhang J and Ding S: The expression of Eg5 predicts a poor outcome for patients with renal cell carcinoma. Med Oncol 30: 476, 2013.

15. Muretta JM, Jun Y, Gross SP, Major J, Thomas DD and Rosenfeld SS: The structural kinetics of switch-1 and the neck linker explain the functions of kinesin-1 and Eg5. Proc Natl Acad Sci USA 112: E6606-E6613, 2015.

16. Ngan ES: Heterogeneity of neuroblastoma. Oncoscience 2: 837-838, 2015.

17. Mei H, Lin ZY and Tong QS: Risk stratification and therapeutics of neuroblastoma: The challenges remain. World J Pediatr 12: 5-7, 2016.

18. Alderton GK: Neuroblastoma: Enhancing risk. Nat Rev Cancer 16: 5, 2016.

19. Exertier P, Javerzat S, Wang B, Franco M, Herbert J, Platonova N, Winandy M, Pujol N, Nivelles O, Ormenese S, et al: Impaired angiogenesis and tumor development by inhibition of the mitotic kinesinEg5. Oncotarget 4: 2302-2316, 2013.

20. Salmela AL and Kallio MJ: Mitosis as an anti-cancer drug target. Chromosoma 122: 431-449, 2013.

21. Sun L, Lu J, Niu Z, Ding K, Bi D, Liu S, Li J, Wu F, Zhang H, Zhao $Z$ and Ding S: A potent chemotherapeutic strategy with Eg5 inhibitor against gemcitabine resistant bladder cancer. PLoS One 10: $\mathrm{e} 0144484,2015$.

22. Good JA, Wang F, Rath O, Kaan HY, Talapatra SK, Podgórski D, MacKay SP and Kozielski F: Optimized S-trityl-L-cysteine-based inhibitors of kinesin spindle protein with potent in vivo antitumor activity in lung cancer xenograftmodels. J Med Chem 56: 1878-1893, 2013

23. Liu G, Xu Z and Hao D: MicroRNA-451 inhibits neuroblastoma proliferation, invasion and migration by targeting macrophage migration inhibitory factor. Mol Med Rep 13: 2253-2260, 2016.

24. Stafman LL and Beierle EA: Cell proliferation in neuroblastoma. Cancers (Basel) 8: E13, 2016.

25. Abualhasan MN, Good JA, Wittayanarakul K, Anthony NG, Berretta G, Rath O, Kozielski F, Sutcliffe OB and Mackay SP: Doing the methylene shuffle-Further insights into the inhibition of mitotic kinesin Eg5 with S-trityl l-cysteine. Eur J Med Chem 54: 483-498, 2012. 
26. Ye XS, Fan L, Van Horn RD, Nakai R, Ohta Y, Akinaga S Murakata C, Yamashita Y, Yin T, Credille KM, et al: A novel Eg5 Inhibitor (LY2523355) causes mitotic arrest and apoptosis in cancer cells and shows potent antitumor activity in xenograft tumor models. Mol Cancer Ther 14: 2463-2472, 2015.

27. Johnson K, Moriarty C, Tania N, Ortman A, DiPietrantonio K, Edens B, Eisenman J, Ok D, Krikorian S, Barragan J, et al: Kif11 dependent cell cycle progression in radial glial cells is required for proper neurogenesis in the zebrafish neural tube. Dev Boil 387: 73-92, 2014.

28. Wiltshire C, Singh BL, Stockley J, Fleming J, Doyle B Barnetson R, Robson CN, Kozielski F and Leung HY: Docetaxel-resistant prostate cancer cells remain sensitive to S-trityl-l-cysteine-Mediated Eg5 inhibition. Mol Cancer Ther 9: 1730-1739, 2010

29. Chen C, Tian F, Lu L, Wang Y, Xiao Z, Yu C and Yu X: Characterization of Cep85-a new antagonist of Nek2A that is involved in the regulation of centrosome disjunction. J Cell Sci 128: 3290-3303, 2015.

30. Bruinsma W, Aprelia M, Kool J, Macurek L, Lindqvist A and Medema RH: Spatial separation of Plk1 phosphorylation and activity. Front Oncol 5: 132, 2015.

31. Smith E, Hégarat N, Vesely C, Roseboom I, Larch C, Streicher H, Straatman K, Flynn H, Skehel M, Hirota T, et al: Differential control of Eg5-dependent centrosome separation by Plk1 and Cdk1. EMBO J 30: 2233-2245, 2011.
32. Cahu J, Olichon A, Hentrich C, Schek H, Drinjakovic J, Zhang C, Doherty-Kirby A, Lajoie G and Surrey T: Phosphorylation by Cdk1 increases the binding of Eg5 to microtubules in vitro and in Xenopus egg extract spindles. PLoS One 3: e3936, 2008.

33. Baas PW and Matamoros AJ: Inhibition of kinesin-5 improves regeneration of injured axons by a novel microtubule-based mechanism. Neural Regen Res 10: 845, 2015.

34. Ou XH, Li S, Xu BZ, Wang ZB, Quan S, Li M, Zhang QH, Ouyang YC, Schatten H, Xing FQ and Sun QY: p38 $\alpha$ MAPK is a MTOC-associated protein regulating spindle assembly, spindle length and accurate chromosome segregation during mouse oocyte meiotic maturation. Cell Cycle 9: 4130-4143, 2010.

35. Srivastava V and Lee H: Synthesis and bio-evaluation of novel quinolino-stilbene derivatives as potential anticancer agents. Bioorg Med Chem 23: 7629-7640, 2015.

36. Gavriilidis P, Giakoustidis A and Giakoustidis D: Aurora kinases and potential medical applications of Aurora kinase inhibitors: A review. J Clin Med Res 7: 742, 2015.

37. Marques S, Fonseca J, Silva PM and Bousbaa H: Targeting the spindle assembly checkpoint for breast cancer treatment. Curr Cancer Drug Targets 15: 272-281, 2015. 